\title{
The classification of flag-transitive Steiner 4-designs
}

\author{
Michael Huber
}

Received: 15 March 2006/ Accepted: 30 November 2006 /

Published online: 10 January 2007

(C) Springer Science + Business Media, LLC 2007

\begin{abstract}
Among the properties of homogeneity of incidence structures flagtransitivity obviously is a particularly important and natural one. Consequently, in the last decades flag-transitive Steiner $t$-designs (i.e. flag-transitive $t$ - $(v, k, 1)$ designs) have been investigated, whereas only by the use of the classification of the finite simple groups has it been possible in recent years to essentially characterize all flag-transitive Steiner 2-designs. However, despite the finite simple group classification, for Steiner $t$-designs with parameters $t>2$ such characterizations have remained challenging open problems for about 40 years (cf. [11, p. 147] and [12, p. 273], but presumably dating back to around 1965). The object of the present paper is to give a complete classification of all flag-transitive Steiner 4-designs. Our result relies on the classification of the finite doubly transitive permutation groups and is a continuation of the author's work [20,21] on the classification of all flag-transitive Steiner 3-designs.
\end{abstract}

Keywords Steiner designs $\cdot$ Flag-transitive group of automorphisms $\cdot$ 2-transitive permutation group

2000 Mathematics Subject Classification. Primary 51E10 - Secondary 05B05 . $20 \mathrm{~B} 25$

\section{Introduction}

For positive integers $t \leq k \leq v$ and $\lambda$, we define a $t-(v, k, \lambda)$ design to be a finite incidence structure $\mathcal{D}=(X, \mathcal{B}, I)$, where $X$ denotes a set of points, $|X|=v$, and $\mathcal{B}$ a set of blocks, $|\mathcal{B}|=b$, with the properties that each block $B \in \mathcal{B}$ is incident with $k$ points, and each $t$-subset of $X$ is incident with $\lambda$ blocks. A flag of $\mathcal{D}$ is an

M. Huber $(\bowtie)$

Mathematisches Institut der Universität Tübingen, Auf der Morgenstelle 10, D-72076 Tübingen, Germany

e-mail: michael.huber@uni-tuebingen.de 
incident point-block pair, that is $x \in X$ and $B \in \mathcal{B}$ such that $(x, B) \in I$. We consider automorphisms of $\mathcal{D}$ as pairs of permutations on $X$ and $\mathcal{B}$ which preserve incidence, and call a group $G \leq \operatorname{Aut}(\mathcal{D})$ of automorphisms of $\mathcal{D}$ flag-transitive (respectively block-transitive, point $t$-transitive) if $G$ acts transitively on the flags (respectively transitively on the blocks, $t$-transitively on the points) of $\mathcal{D}$. For short, $\mathcal{D}$ is said to be, e.g., flag-transitive if $\mathcal{D}$ admits a flag-transitive group of automorphisms.

For historical reasons, a $t-(v, k, \lambda)$ design with $\lambda=1$ is called a Steiner $t$-design (sometimes this is also known as a Steiner system). We note that in this case each block is determined by the set of points which are incident with it, and thus can be identified with a $k$-subset of $X$ in a unique way. If $t<k<v$ holds, then we speak of a non-trivial Steiner $t$-design.

Among the properties of homogeneity of incidence structures flagtransitivity obviously is a particularly important and natural one. Consequently, in the last decades flag-transitive Steiner $t$-designs have been investigated, in particular for the case $t=2$. The general study of flag-transitive Steiner 2-designs was introduced by D.G. Higman and J.E. McLaughlin [19] proving that a flag-transitive group $G \leq \operatorname{Aut}(\mathcal{D})$ of automorphisms of a Steiner 2-design $\mathcal{D}$ is necessarily primitive on the points of $\mathcal{D}$. They posed the problem of classifying all finite flag-transitive projective planes, and showed that such planes are desarguesian if its orders are suitably restricted. Much later W.M. Kantor [27] determined all such planes apart from the still open case when the group of automorphisms is a Frobenius group of prime degree (cf. [5, Section 1 and 2], [25], and [28] for a detailed survey on flag-transitive planes). In a big common effort, F. Buekenhout, A. Delandtsheer, J. Doyen, P.B. Kleidman, M.W. Liebeck, and J. Saxl [4, 13, 30, 32, 35] essentially characterized all finite flag-transitive linear spaces, that is flag-transitive Steiner 2-designs (for the incomplete case with a 1-dimensional affine group of automorphisms, see [4, Section 4] and [28, Section 3]). Their result, which was announced in 1990, makes use of the classification of the finite simple groups.

However, despite the classification of the finite simple groups, for Steiner $t$-designs with parameters $t>2$ such characterizations have remained challenging open problems for about 40 years (see [11, p. 147] and [12, p. 273], but presumably dating back to around 1965 , cf. [33, 37]). Recently, the author [20,21] completely determined all flag-transitive Steiner 3-designs using the classification of the finite doubly transitive permutation groups, which in turn relies on the classification of the finite simple groups.

The object of the present paper is to give a complete classification of all flagtransitive Steiner 4-designs. Our approach uses again the classification of the finite doubly transitive permutation groups.

The classification of all non-trivial Steiner 4-designs admitting a flag-transitive group of automorphisms can be stated as follows.

Main Theorem. Let $\mathcal{D}=(X, \mathcal{B}, I)$ be a non-trivial Steiner 4-design. Then $G \leq \operatorname{Aut}(\mathcal{D})$ acts flag-transitively on $\mathcal{D}$ if and only if one of the following occurs:

(1) $\mathcal{D}$ is isomorphic to the Witt 4-(11,5,1) design, and $G \cong M_{11}$,

(2) $\mathcal{D}$ is isomorphic to the Witt $4-(23,7,1)$ design, and $G \cong M_{23}$.

For a detailed description of the Witt $t-(v, k, 1)$ designs with their associated Mathieu groups $M_{v}$ of degree $v$, we refer, e.g., to [39]. 


\section{Definitions and preliminary results}

If $\mathcal{D}=(X, \mathcal{B}, I)$ is a $t$ - $(v, k, \lambda)$ design with $t \geq 2$, and $x \in X$ arbitrary, then the derived design with respect to $x$ is $\mathcal{D}_{x}=\left(X_{x}, \mathcal{B}_{x}, I_{x}\right)$, where $X_{x}=X \backslash\{x\}$, $\mathcal{B}_{x}=\{B \in \mathcal{B}:(x, B) \in I\}$ and $I_{x}=\left.I\right|_{X_{x} \times \mathcal{B}_{x}}$. In this case, $\mathcal{D}$ is also called an $e x$ tension of $\mathcal{D}_{x}$. Obviously, $\mathcal{D}_{x}$ is a $(t-1)-(v-1, k-1, \lambda)$ design.

Let $G$ be a permutation group on a non-empty set $X$. For $g \in G$, let $\operatorname{Fix}_{X}(g)$ denote the set of fixed points of $g$ in $X$. We call $G$ semi-regular if the identity is the only element that fixes any point of $X$. If additionally $G$ is transitive, then it is said to be regular. Furthermore, for $x \in X$, the orbit $x^{G}$ containing $x$ is called regular if it has length $|G|$. If $\left\{x_{1}, \ldots, x_{m}\right\} \subseteq X$, let $G_{\left\{x_{1}, \ldots, x_{m}\right\}}$ be its setwise stabilizer and $G_{x_{1}, \ldots, x_{m}}$ its pointwise stabilizer.

For $\mathcal{D}=(X, \mathcal{B}, I)$ a Steiner $t$-design with $G \leq \operatorname{Aut}(\mathcal{D})$, let $G_{B}$ denote the setwise stabilizer of a block $B \in \mathcal{B}$, and for $x \in X$, we define $G_{x B}=G_{x} \cap G_{B}$.

Let $\mathbb{Z}^{+}$be the set of positive integers (without 0 ). For integers $m$ and $n$, let $(m, n)$ denote the greatest common divisor of $m$ and $n$, and we write $m \mid n$ if $m$ divides $n$.

For any $x \in \mathbb{R}$, let $\lfloor x\rfloor$ denote the greatest positive integer which is at most $x$.

All other notation is standard.

The approach to the classification of all flag-transitive Steiner 4-designs starts with the following proposition which can be deduced from a result of R.E. Block [3, Thm. 2].

Proposition 1 (cf. $[6,21])$. Let $\mathcal{D}=(X, \mathcal{B}, I)$ be a Steiner $t$-design with $t \geq 3$. If $G \leq \operatorname{Aut}(\mathcal{D})$ acts flag-transitively on $\mathcal{D}$, then $G$ also acts point 2-transitively on $\mathcal{D}$.

We note that if $t=2$, then it is elementary that conversely the point 2-transitivity of $G \leq \operatorname{Aut}(\mathcal{D})$ implies its flag-transitivity.

The above result allows us to make use of the classification of all finite 2-transitive permutation groups, which itself relies on the classification of all finite simple groups (cf. $[10,16,17,18,23,26,31,34]$ ).

The list of groups is as follows.

Let $G$ be a finite 2-transitive permutation group on a non-empty set $X$. Then $G$ is either of

(A) Affine Type: $G$ contains a regular normal subgroup $T$ which is elementary Abelian of order $v=p^{d}$, where $p$ is a prime. If $a$ divides $d$, and if we identify $G$ with a group of affine transformations

$$
x \mapsto x^{g}+u
$$

of $V=V(d, p)$, where $g \in G_{0}$ and $u \in V$, then particularly one of the following occurs:

(1) $G \leq A \Gamma L\left(1, p^{d}\right)$

(2) $G_{0} \unrhd S L\left(\frac{d}{a}, p^{a}\right), d \geq 2 a$

(3) $G_{0} \unrhd \operatorname{Sp}\left(\frac{2 d}{a}, p^{a}\right), d \geq 2 a$

(4) $G_{0} \unrhd G_{2}\left(2^{a}\right)^{\prime}, d=6 a$

(5) $G_{0} \cong A_{6}$ or $A_{7}, v=2^{4}$

(6) $G_{0} \unrhd S L(2,3)$ or $S L(2,5), v=p^{2}, p=5,7,11,19,23,29$ or 59 , or $v=3^{4}$ 
(7) $G_{0}$ contains a normal extraspecial subgroup $E$ of order $2^{5}$, and $G_{0} / E$ is isomorphic to a subgroup of $S_{5}, v=3^{4}$

(8) $G_{0} \cong S L(2,13), v=3^{6}$,

or

(B) Almost Simple Type: $G$ contains a simple normal subgroup $N$, and $N \leq G \leq \operatorname{Aut}(N)$. In particular, one of the following holds, where $N$ and $v=|X|$ are given as follows:

(1) $A_{v}, v \geq 5$

(2) $\operatorname{PSL}(d, q), d \geq 2, v=\frac{q^{d}-1}{q-1}$, where $(d, q) \neq(2,2),(2,3)$

(3) $\operatorname{PSU}\left(3, q^{2}\right), v=q^{3}+1, q>2$

(4) $S z(q), v=q^{2}+1, q=2^{2 e+1}>2$

(Suzuki groups)

(5) $\operatorname{Re}(q), v=q^{3}+1, q=3^{2 e+1}>3$

(Ree groups)

(6) $\operatorname{Sp}(2 d, 2), d \geq 3, v=2^{2 d-1} \pm 2^{d-1}$

(7) $\operatorname{PSL}(2,11), v=11$

(8) $\operatorname{PSL}(2,8), v=28$ ( $N$ is not 2-transitive)

(9) $M_{v}, v=11,12,22,23,24$

(Mathieu groups)

(10) $M_{11}, v=12$

(11) $A_{7}, v=15$

(12) $H S, v=176$

(Higman-Sims group)

(13) $\mathrm{Co}_{3}, v=276$.

(smallest Conway group)

For required basic properties of the listed groups, we refer, e.g., to [9, 24, 29, 36, 38].

We will now indicate some helpful combinatorial tools on which we rely in the sequel. Let $r$ (respectively $\lambda_{2}$ ) denote the total number of blocks incident with a given point (respectively pair of distinct points), and let all further parameters be as defined at the beginning of Section 1 .

Obvious is the subsequent fact.

Lemma 2. Let $\mathcal{D}=(X, \mathcal{B}, I)$ be a Steiner $t$-design. If $G \leq \operatorname{Aut}(\mathcal{D})$ acts flagtransitively on $\mathcal{D}$, then, for any $x \in X$, the division property

$$
r|| G_{x} \mid
$$

holds.

Elementary counting arguments give the following standard assertions.

Lemma 3. Let $\mathcal{D}=(X, \mathcal{B}, I)$ be a $t-(v, k, \lambda)$ design. Then the following holds:

(a) $b k=v r$.

(b) $\left(\begin{array}{l}v \\ t\end{array}\right) \lambda=b\left(\begin{array}{l}k \\ t\end{array}\right)$.

$\underline{\text { Springer }}$ 
(c) $r(k-1)=\lambda_{2}(v-1)$ for $t \geq 2$, where $\lambda_{2}=\lambda \frac{\left(\begin{array}{c}v-2 \\ t-2\end{array}\right)}{\left(\begin{array}{c}k-2 \\ t-2\end{array}\right)}$.

(d) In particular, if $t=4$, then $(k-2)(k-3) \mid(v-2)(v-3)$.

For non-trivial Steiner $t$-designs lower bounds for $v$ in terms of $k$ and $t$ are known.

Proposition 4 (cf. $[8,37])$. If $\mathcal{D}=(X, \mathcal{B}, I)$ is a non-trivial Steinert-design, then the following holds:

(a) $v \geq(t+1)(k-t+1)$.

(b) $v-t+1 \geq(k-t+2)(k-t+1)$ for $t>2$. If equality holds, then $(t, k, v)=$ $(3,4,8),(3,6,22),(3,12,112),(4,7,23)$, or $(5,8,24)$.

We note that (a) is stronger for $k<2(t-1)$, while (b) is stronger for $k>2(t-1)$. For $k=2(t-1)$ both assert that $v \geq t^{2}-1$.

As we are in particular interested in the case when $t=4$, we deduce from (b) the following upper bound for the positive integer $k$.

Corollary 5. Let $\mathcal{D}=(X, \mathcal{B}, I)$ be a non-trivial Steiner 4-design. Then

$$
k \leq\left\lfloor\sqrt{v}+\frac{5}{2}\right\rfloor .
$$

Remark 6. If $G \leq \operatorname{Aut}(\mathcal{D})$ acts flag-transitively on any Steiner 4-design $\mathcal{D}$, then applying Proposition 1 and Lemma 3 (b) yields the equation

$$
b=\frac{v(v-1)(v-2)(v-3)}{k(k-1)(k-2)(k-3)}=\frac{v(v-1)\left|G_{x y}\right|}{\left|G_{B}\right|},
$$

where $x$ and $y$ are two distinct points in $X$ and $B$ is a block in $\mathcal{B}$, and thus

$$
(v-2)(v-3)=(k-1)(k-2)(k-3) \frac{\left|G_{x y}\right|}{\left|G_{x B}\right|} \quad \text { if } x \in B .
$$

Finally, we assert some lemmas which will be required in Section 3.2 of the proof of the Main Theorem. Let $q$ be a prime power $p^{e}$, and $U$ a subgroup of $P S L(2, q)$. Furthermore, let $N_{l}$ denote the number of orbits of length $l$ and let $n=(2, q-1)$. In [22, Ch.5], we have in particular determined the orbit-lengths from the action of subgroups of $\operatorname{PSL}(2, q)$ on the points of the projective line. For the list of subgroups of $\operatorname{PSL}(2, q)$, we thereby refer to [15, Ch. 12, p. 285f.] or [24, Ch. II, Thm. 8.27].

Lemma 7. Let $U$ be the cyclic group of order $c$ with $c \mid \frac{q \pm 1}{n}$. Then, we have (a) if $c \mid \frac{q+1}{n}$, then $N_{c}=(q+1) / c$, 
(b) if $c \mid \frac{q-1}{n}$, then $N_{1}=2$ and $N_{c}=(q-1) / c$.

Lemma 8. Let $U$ be the dihedral group of order $2 c$ with $c \mid \frac{q \pm 1}{n}$. Then

(i) for $q \equiv 1(\bmod 4)$, we have

(a) if $c \mid \frac{q+1}{2}$, then $N_{c}=2$ and $N_{2 c}=(q+1-2 c) /(2 c)$,

(b) if $c \mid \frac{q-1}{2}$, then $N_{2}=1, N_{c}=2$, and $N_{2 c}=(q-1-2 c) /(2 c)$, unless $c=2$, in which case $N_{2}=3$ and $N_{4}=(q-5) / 4$,

(ii) for $q \equiv 3(\bmod 4)$, we have

(a) if $c \mid \frac{q+1}{2}$, then $N_{2 c}=(q+1) /(2 c)$,

(b) if $c \mid \frac{q-1}{2}$, then $N_{2}=1$ and $N_{2 c}=(q-1) /(2 c)$,

(iii) for $q \equiv 0(\bmod 2)$, we have

(a) if $c \mid q+1$, then $N_{c}=1$ and $N_{2 c}=(q+1-c) /(2 c)$,

(b) if $c \mid q-1$, then $N_{2}=1, N_{c}=1$, and $N_{2 c}=(q-1-c) /(2 c)$.

Lemma 9. Let $U$ be the elementary Abelian group of order $\bar{q} \mid q$. Then, we have $N_{1}=1$ and $N_{\bar{q}}=q / \bar{q}$.

Lemma 10. Let $U$ be a semi-direct product of the elementary Abelian group of order $\bar{q} \mid q$ and the cyclic group of order $c$ with $c \mid \bar{q}-1$ and $c \mid q-1$. Then, we have $N_{1}=1, N_{\bar{q}}=1$, and $N_{c \bar{q}}=(q-\bar{q}) /(c \bar{q})$.

Lemma 11. Let $U$ be $\operatorname{PSL}(2, \bar{q})$ with $\bar{q}^{m}=q, m \geq 1$. Then, we have $N_{\bar{q}+1}=1$, $N_{\bar{q}(\bar{q}-1)}=1$ if $m$ is even, and all other orbits are regular.

Lemma 12. Let $U$ be $\operatorname{PGL}(2, \bar{q})$ with $\bar{q}^{m}=q, m>1$ even. Then, we have $N_{\bar{q}+1}=1$, $N_{\bar{q}(\bar{q}-1)}=1$, and all other orbits are regular.

Lemma 13. Let $U$ be isomorphic to $A_{4}$. Then

(i) for $q \equiv 1(\bmod 4)$, we have

(a) if $3 \mid \frac{q+1}{2}$, then $N_{6}=1$ and $N_{12}=(q-5) / 12$,

(b) if $3 \mid \frac{q-1}{2}$, then $N_{4}=2, N_{6}=1$, and $N_{12}=(q-13) / 12$,

(c) if $3 \mid q$, then $N_{4}=1, N_{6}=1$, and $N_{12}=(q-9) / 12$,

(ii) for $q \equiv 3(\bmod 4)$, we have

(a) if $3 \mid \frac{q+1}{2}$, then $N_{12}=(q+1) / 12$,

(b) if $3 \mid \frac{q-1}{2}$, then $N_{4}=2$ and $N_{12}=(q-7) / 12$,

(c) if $3 \mid q$, then $N_{4}=1$ and $N_{12}=(q-3) / 12$,

(iii) for $q=2^{e}, e \equiv 0(\bmod 2)$, we have $N_{1}=1, N_{4}=1$, and $N_{12}=(q-4) / 12$. 
Lemma 14. Let $U$ be isomorphic to $S_{4}$. Then

(i) for $q \equiv 1(\bmod 8)$, we have

(a) if $3 \mid \frac{q+1}{2}$, then $N_{6}=1, N_{12}=1$, and $N_{24}=(q-17) / 24$,

(b) if $3 \mid \frac{q-1}{2}$, then $N_{6}=1, N_{8}=1, N_{12}=1$, and $N_{24}=(q-25) / 24$,

(c) if $3 \mid q$, then $N_{4}=1, N_{6}=1$, and $N_{24}=(q-9) / 24$,

(ii) for $q \equiv-1(\bmod 8)$, we have

(a) if $3 \mid \frac{q+1}{2}$, then $N_{24}=(q+1) / 24$,

(b) if $3 \mid \frac{q-1}{2}$, then $N_{8}=1$ and $N_{24}=(q-7) / 24$.

Lemma 15. Let $U$ be isomorphic to $A_{5}$. Then

(i) for $q \equiv 1(\bmod 4)$, we have

(a) if $q=5^{e}, e \equiv 1(\bmod 2)$, then $N_{6}=1$ and $N_{60}=(q-5) / 60$,

(b) if $q=5^{e}, e \equiv 0(\bmod 2)$, then $N_{6}=1, N_{20}=1$, and $N_{60}=(q-25) / 60$,

(c) if $15 \mid \frac{q+1}{2}$, then $N_{30}=1$ and $N_{60}=(q-29) / 60$,

(d) if $3 \mid \frac{q+1}{2}$ and $5 \mid \frac{q-1}{2}$, then $N_{12}=1, N_{30}=1$, and $N_{60}=(q-41) / 60$,

(e) if $3 \mid \frac{q-1}{2}$ and $5 \mid \frac{q+1}{2}$, then $N_{20}=1, N_{30}=1$, and $N_{60}=(q-49) / 60$,

(f) if $15 \mid \frac{q-1}{2}$, then $N_{12}=1, N_{20}=1, N_{30}=1$, and $N_{60}=(q-61) / 60$,

(g) if $3 \mid q$ and $5 \mid \frac{q+1}{2}$, then $N_{10}=1$ and $N_{60}=(q-9) / 60$,

(h) if $3 \mid q$ and $5 \mid \frac{q-1}{2}$, then $N_{10}=1, N_{12}=1$, and $N_{60}=(q-21) / 60$,

(ii) for $q \equiv 3(\bmod 4)$, we have

(a) if $15 \mid \frac{q+1}{2}$, then $N_{60}=(q+1) / 60$,

(b) if $3 \mid \frac{q+1}{2}$ and $5 \mid \frac{q-1}{2}$, then $N_{12}=1$ and $N_{60}=(q-11) / 60$,

(c) if $3 \mid \frac{q-1}{2}$ and $5 \mid \frac{q+1}{2}$, then $N_{20}=1$ and $N_{60}=(q-19) / 60$,

(d) if $15 \mid \frac{q-1}{2}$, then $N_{12}=1, N_{20}=1$, and $N_{60}=(q-31) / 60$.

\section{Proof of the Main Theorem}

Using the notation as before, let $\mathcal{D}=(X, \mathcal{B}, I)$ be a non-trivial Steiner 4-design with $G \leq \operatorname{Aut}(\mathcal{D})$ acting flag-transitively on $\mathcal{D}$ throughout the proof. We recall that due to Proposition 1, we may restrict ourselves to the consideration of the finite 2-transitive permutation groups listed in Section 2. Clearly, in the following we may assume that $k>4$ as trivial Steiner 4-designs are excluded. For each of the Cases (A)(5)-(8) and (B)(8), (11)-(13) we have only a small number of possibilities for $k$ to check, which can easily be ruled out by hand using Lemma 2, Lemma 3(b)-(d), and Corollary 5. 


\subsection{Groups of automorphisms of affine type}

We first assume that $G$ is of affine type.

Case $G \leq A \Gamma L(1, v), v=p^{d}$.

As $G$ is point 2-transitive, we have $|G|=v(v-1) a$ with $a \mid d$. Using Lemma 2, we obtain

$$
\left(p^{d}-2\right)\left(p^{d}-3\right)|a(k-1)(k-2)(k-3)| d(k-1)(k-2)(k-3),
$$

and hence in particular

$$
\left(p^{d}-2\right)\left(p^{d}-3\right) \leq d(k-1)(k-2)(k-3) .
$$

But, Proposition 4(b) yields

$$
p^{d}-3 \geq(k-2)(k-3),
$$

and thus

$$
p^{d}-2 \leq d(k-1) \text {. }
$$

With regard to Corollary 5, this leaves only a very small number of possibilities for $k$ to check, which can easily be ruled out by hand using Lemma 3(b) and (c). Therefore, $G \leq \operatorname{Aut}(\mathcal{D})$ cannot act flag-transitively on any non-trivial Steiner 4-design $\mathcal{D}$.

Case $G_{0} \unrhd S L\left(\frac{d}{a}, p^{a}\right), d \geq 2 a$.

In the following, let $e_{i}$ denote the $i$-th basis vector of the vector space $V=V\left(\frac{d}{a}, p^{a}\right)$, and $\left\langle e_{i}\right\rangle$ the 1-dimensional vector subspace spanned by $e_{i}$.

First, let $p^{a}>3$. For $d=2 a$, let $U=U\left(\left\langle e_{1}\right\rangle\right) \leq G_{0}$ denote the subgroup of all transvections with axis $\left\langle e_{1}\right\rangle$. Then $U$ consists of all elements of the form

$$
\left(\begin{array}{ll}
1 & 0 \\
c & 1
\end{array}\right), \quad c \in G F\left(p^{a}\right) \text { arbitrary. }
$$

Clearly, $U$ fixes as points only the elements of $\left\langle e_{1}\right\rangle$. Thus, $G_{0}$ has point-orbits of length at least $p^{a}$ outside $\left\langle e_{1}\right\rangle$. Let $S=\left\{0, e_{1}, x, y\right\}$ be a 4-subset of distinct points with $x, y \in\left\langle e_{1}\right\rangle$. Obviously, $U$ fixes the unique block $B \in \mathcal{B}$ which is incident with $S$. If $B$ contains at least one point outside $\left\langle e_{1}\right\rangle$, then we would obtain $k \geq p^{a}+4$, which is not possible as $k \leq p^{a}+2$ in view of Corollary 5. Thus $B$ is contained completely in $\left\langle e_{1}\right\rangle$, and as $G$ is flag-transitive, we conclude that each block lies in an affine line. But, by the definition of Steiner 4-designs, any four distinct non-collinear points must also be incident with a unique block, a contradiction.

For $d \geq 3 a, S L\left(\frac{d}{a}, p^{a}\right)_{e_{1}}$ and hence also $G_{0, e_{1}}$ acts point-transitively on $V \backslash\left\langle e_{1}\right\rangle$. Again, let $S=\left\{0, e_{1}, x, y\right\}$ be a 4-subset of distinct points with $x, y \in\left\langle e_{1}\right\rangle$. If the unique block $B \in \mathcal{B}$ which is incident with $S$ contains some point outside $\left\langle e_{1}\right\rangle$, then it would already contain all points outside, thus at least $p^{d}-p^{a}+4$ many, which 
obviously contradicts Corollary 5 . We conclude that $B$ lies completely in $\left\langle e_{1}\right\rangle$, and may proceed as above.

Now, let $p^{a}=2$. Then $v=2^{d}$. For $d=3$, we have $v=8$ and $k=5$ by Corollary 5 , which is not possible in view of Lemma 3 (c). Therefore, we assume that $d>3$. We remark that clearly any three distinct points are non-collinear in $A G(d, 2)$ and hence define an affine plane. Let $\mathcal{E}=\left\langle e_{1}, e_{2}\right\rangle$ denote the 2-dimensional vector subspace spanned by $e_{1}$ and $e_{2}$. Then $S L(d, 2)_{\mathcal{E}}$ and hence also $G_{0, \mathcal{E}}$ acts point-transitively on $V \backslash \mathcal{E}$. If the unique block $B \in \mathcal{B}$ which is incident with the 4-subset $\left\{0, e_{1}, e_{2}, e_{1}+e_{2}\right\}$ contains some point outside $\mathcal{E}$, then it would already contain all points of $V \backslash \mathcal{E}$, and hence $k \geq 2^{d}-4+4=2^{d}$, a contradiction to Corollary 5. Therefore, $B$ can be identified with $\mathcal{E}$, and the flag-transitivity of $G$ implies that each block must be an affine plane, a contradiction as $k>4$. Similar arguments hold for $p^{a}=3$.

Case $G_{0} \unrhd \operatorname{Sp}\left(\frac{2 d}{a}, p^{a}\right), d \geq 2 a$.

First, let $p^{a} \neq 2$. The permutation group $P \operatorname{Sp}\left(\frac{2 d}{a}, p^{a}\right)$ on the points of the associated projective space is a rank 3 group, and the orbits of the one-point stabilizer are known (e.g. [24, Ch. II, Thm.9.15(b)]). Thus, $G_{0} \unrhd S p\left(\frac{2 d}{a}, p^{a}\right)$ has exactly two orbits on $V \backslash\langle x\rangle(0 \neq x \in V)$ of length at least

$$
\frac{p^{a}\left(p^{2 d-2 a}-1\right)}{p^{a}-1}=\sum_{i=1}^{\frac{2 d}{a}-2} p^{i a}>p^{d} .
$$

Let $S=\{0, x, y, z\}$ be a 4-subset with $y, z \in\langle x\rangle$. If the unique block which is incident with $S$ contains at least one point of $V \backslash\langle x\rangle$, then we would have $k>p^{d}+4$, which is impossible since $k \leq p^{d}+2$ by Corollary 5 . Therefore, we can argue as in the previous Case.

Now, let $p^{a}=2$. Then $v=2^{2 d}$. For $d=2$ (here $\operatorname{Sp}(4,2) \cong S_{6}$ as well-known), Corollary 5 yields $k \leq 6$. But, Lemma 3 (d) rules out the cases when $k=5$ or 6 . Thus, let $d>2$. It is easily seen that there are $2^{2 d-1}\left(2^{2 d}-1\right)$ hyperbolic pairs in the non-degenerate symplectic space $V=V(2 d, 2)$, and by Witt's theorem, $\operatorname{Sp}(2 d, 2)$ is transitive on these hyperbolic pairs. Let $\{x, y\}$ denote a hyperbolic pair, and $\mathcal{E}=\langle x, y\rangle$ the hyperbolic plane spanned by $\{x, y\}$. As $\mathcal{E}$ is non-degenerate, we have the orthogonal decomposition

$$
V=\mathcal{E} \perp \mathcal{E}^{\perp}
$$

Obviously, $\operatorname{Sp}(2 d, 2)_{\{x, y\}}$ stabilizes $\mathcal{E}^{\perp}$ as a subspace, which implies that $\operatorname{Sp}(2 d, 2)_{\{x, y\}} \cong \operatorname{Sp}(2 d-2,2)$. As Out $(\operatorname{Sp}(2 d, 2))=1$, we have therefore

$$
\operatorname{Sp}(2 d-2,2) \cong \operatorname{Sp}(2 d, 2)_{\{x, y\}} \unlhd \operatorname{Sp}(2 d, 2)_{\mathcal{E}}=G_{0, \mathcal{E}} .
$$

As $\operatorname{Sp}(2 d-2,2)$ acts transitively on the non-zero vectors of the $(2 d-2)$-dimensional symplectic subspace, the smallest orbit on $V \backslash \mathcal{E}$ under $G_{0, \mathcal{E}}$ has length at least $2^{2 d-2}-1$. If the unique block $B \in \mathcal{B}$ which is incident with the 4-subset $\{0, x, y, x+y\}$ contains some point in $V \backslash \mathcal{E}$, then we would have $k \geq 2^{2 d-2}+3$, a contradiction to Corollary 5 . Thus $B$ can be identified with $\mathcal{E}$, and by the flag-transitivity of $G$, each block must be an affine plane, yielding a contradiction. 
Case $G_{0} \unrhd G_{2}\left(2^{a}\right)^{\prime}, d=6 a$.

First, let $a=1$. Then $v=2^{6}=64$ and $k \leq 10$ by Corollary 5. On the other hand, we have $\left|G_{2}(2)^{\prime}\right|=2^{5} \cdot 3^{3} \cdot 7$ and $\left|\operatorname{Out}\left(G_{2}(2)^{\prime}\right)\right|=2$. In view of Lemma 2 this gives

$$
r=\frac{63 \cdot 62 \cdot 61}{(k-1)(k-2)(k-3)}|| G_{0}|| 2^{6} \cdot 3^{3} \cdot 7
$$

which implies that $k$ is at least 63 , a contradiction.

Now, let $a>1$. As here $G_{2}\left(2^{a}\right)$ is simple non-Abelian, it is sufficient to consider $G_{0} \unrhd G_{2}\left(2^{a}\right)$. The permutation group $G_{2}\left(2^{a}\right)$ is of rank 4 , and for $0 \neq x \in V$ the onepoint stabilizer $G_{2}\left(2^{a}\right)_{x}$ has exactly three orbits $\mathcal{O}_{i}(i=1,2,3)$ on $V \backslash\langle x\rangle$ of length $2^{3 a}-2^{a}, 2^{5 a}-2^{3 a}, 2^{6 a}-2^{5 a}$ (cf., e.g., [1] or [7, Thm. 3.1]). Thus, $G_{0}$ has exactly three orbits on $V \backslash\langle x\rangle$ of length at least $\left|\mathcal{O}_{i}\right|$. Let $S=\{0, x, y, z\}$ be a 4-subset with $y, z \in\langle x\rangle$. If the unique block $B \in \mathcal{B}$ which is incident with $S$ contains at least one point of $V \backslash\langle x\rangle$ in $\mathcal{O}_{2}$ or $\mathcal{O}_{3}$, then we would obtain as above a contradiction to Corollary 5. Thus, we only have to consider the case when $B$ contains points of $V \backslash\langle x\rangle$ which all lie in $\mathcal{O}_{1}$. By [1], the orbit $\mathcal{O}_{1}$ is exactly known, and we have

$$
\mathcal{O}_{1}=x \Delta \backslash\langle x\rangle
$$

where $x \Delta=\{y \in V \mid f(x, y, z)=0$ for all $z \in V\}$ with an alternating trilinear form $f$ on $V$. Then $B$ consists, apart from elements of $\langle x\rangle$, exactly of $\mathcal{O}_{1}$. Since $\left|\mathcal{O}_{1}\right| \neq 1$, we can choose $\langle\bar{x}\rangle \in x \Delta$ with $\langle\bar{x}\rangle \neq\langle x\rangle$. But then, for symmetric reasons, the 4-subset $\{0, \bar{x}, \bar{y}, \bar{z}\}$ with $\bar{y}, \bar{z} \in\langle\bar{x}\rangle$ must also be incident with the unique block $B$, a contradiction to the fact that $\bar{x} \Delta \neq x \Delta$ for $\langle\bar{x}\rangle \neq\langle x\rangle$. Consequently, $B$ is contained completely in $\langle x\rangle$, and we may argue as in the Cases above.

\subsection{Groups of automorphisms of almost simple type}

We consider now successively those cases where $G$ is of almost simple type.

Case $N=A_{v}, v \geq 5$. We may assume that $v \geq 6$, and then $A_{v}$ and hence also $G$ is 4-transitive and does not act on any non-trivial Steiner 4-design $\mathcal{D}$ in view of [26, Thm. 3].

Case $N=\operatorname{PSL}(d, q), d \geq 2, v=\frac{q^{d}-1}{q-1}$, where $(d, q) \neq(2,2),(2,3)$.

We distinguish two subcases:

Subcase $N=\operatorname{PSL}(2, q), v=q+1, q=p^{e}>3$.

Here $\operatorname{Aut}(N)=P \Gamma L(2, q)$, and $|G|=(q+1) q \frac{(q-1)}{n} a$ with $n=(2, q-1)$ and $a \mid$ ne. We may assume that $q \geq 5$.

First, we assume that $N=G$. Then, by Remark 6, we obtain

$$
(q-2)\left|P S L(2, q)_{0 B}\right| \cdot n=(k-1)(k-2)(k-3)
$$

which is equivalent to

$$
(q-2)\left|P S L(2, q)_{0 B}\right| \cdot n+6=k\left(k^{2}-6 k+11\right) .
$$


Thus, we have in particular

$$
k|(q-2)| P S L(2, q)_{0 B} \mid \cdot n+6 .
$$

Since $\operatorname{PSL}(2, q)_{B}$ acts transitively on the points of $B$, we have

$$
k=\left|0^{P S L(2, q)_{B}}\right|=\left|P S L(2, q)_{B}: P S L(2, q)_{0 B}\right| .
$$

Let us first consider the case (1.1) when $\left|P S L(2, q)_{0 B}\right|=1$. a) If $q$ is even, then $k \mid q+4$ by property (3). On the other hand, using Eq. (4), we have $k=\left|P S L(2, q)_{B}\right| \mid$ $|P S L(2, q)|=q^{3}-q$. As

$\left(q^{3}-q, q+4\right)=(60, q+4)=4 \cdot\left(15,2^{e-2}+1\right)= \begin{cases}4, & \text { if } e \text { is even and } 4 \nmid e \\ 4 \cdot 3, & \text { if } e \text { is odd } \\ 4 \cdot 5, & \text { if } 4 \mid e\end{cases}$

the possible values for $k$ can immediately be ruled out by hand using Eq. (1).

b) If $q$ is odd, we have $k=\left|P S L(2, q)_{B}\right| \mid 2(q+1)$ due to property (3) and Eq. (4). Examining the list of subgroups of $P S L(2, q)$ (cf. [15, Ch. 12, p. 285f.] or [24, Ch. II, Thm. 8.27]), we have to consider the following possibilities:

(1.1.b) (i) $\operatorname{PSL}(2, q)_{B}$ is conjugate to a cyclic subgroup of order $c$ with $c \mid \frac{q+1}{2}$ of $\operatorname{PSL}(2, q)$, and $k=c$.

(1.1.b) (ii) $\operatorname{PSL}(2, q)_{B}$ is conjugate to a dihedral subgroup of order $2 c$ with $c \mid \frac{q+1}{2}$ of $P S L(2, q)$, and $k=2 c$.

(1.1.b) (iii) $P S L(2, q)_{B}$ is conjugate to $A_{4}$, and $k=12$.

(1.1.b) (iv) $P S L(2, q)_{B}$ is conjugate to $S_{4}$, and $k=24$.

(1.1.b) (v) $\operatorname{PSL}(2, q)_{B}$ is conjugate to $A_{5}$, and $k=60$.

(1.1.b)(i): By Eq. (1), we have

$$
c \mid \frac{q+1}{2}=\frac{(c-1)(c-2)(c-3)+6}{4}=\frac{c\left(c^{2}-6 c+11\right)}{4} .
$$

Since 4 does not divide $c^{2}-6 c+11$, this is impossible.

(1.1.b)(ii): Using Eq. (1), we obtain

$$
c \mid \frac{q+1}{2}=\frac{(2 c-1)(c-1)(2 c-3)+3}{2},
$$

which is not possible as $(2 c-1)(c-1)(2 c-3)+3=4 c^{3}-12 c^{2}+11 c \equiv c(\bmod$ $2 c)$.

(1.1.b)(iii)-(v): For each given value of $k$, Eq. (1) gives in each subcase that $q$ is not a prime power.

We consider now the case (1.2) when $\left|P S L(2, q)_{0 B}\right|=2$. a) If $q$ is even, then we have $k=\frac{\left|P S L(2, q)_{B}\right|}{2} \mid 2(q+1)$ due to property (3) and Eq. (4). Considering the list of subgroups of $\operatorname{PSL}(2, q)$, we have the following possibilities: 
(1.2.a) (i) $P S L(2, q)_{B}$ is conjugate to a cyclic subgroup of order $c$ with $c \mid q+1$ of $\operatorname{PSL}(2, q)$, and $k=\frac{c}{2}$.

(1.2.a) (ii) $\operatorname{PSL}(2, q)_{B}$ is conjugate to a dihedral subgroup of order $2 c$ with $c \mid q+1$ of $\operatorname{PSL}(2, q)$, and $k=c$.

(1.2.a) (iii) $\operatorname{PSL}(2, q)_{B}$ is conjugate to $\operatorname{PSL}(2, \bar{q})$ with $\bar{q} \mid 4$, and $k=30$.

(1.2.a) (iv) $P S L(2, q)_{B}$ is conjugate to $A_{4}$, and $k=6$.

(1.2.a)(i),(iii),(iv): In view of Lemmas 7, 12, respectively 13(iii), clearly $k$ cannot take the given values.

(1.2.a)(ii): Considering Eq. (1), for $k=c>4$ clearly the right hand side of the equation is divisible by 8 , but not the left hand side.

b) If $q$ is odd, then $k \mid 2(2 q-1)$ by property (3). On the other hand, Eq. (4) yields $k=\frac{\left|P S L(2, q)_{B}\right|}{2} \mid \frac{|P S L(2, q)|}{2}=\frac{q^{3}-q}{4}$. Since $\left(\frac{q^{3}-q}{4}, 2(2 q-1)\right)=2 \cdot\left(\frac{q^{3}-q}{8}, 2 q-\right.$ $1)=2 \cdot(3, q+1)$ only $k=6$ can occur, and Eq. (1) gives then $q=17$. However, it is known that there does not exist any $4-(18,6,1)$ design (cf. [40, Thm. 6]).

Finally, let us consider the case (1.3) when $\left|P S L(2, q)_{0 B}\right|>2$. Examining the list of subgroups of $\operatorname{PSL}(2, q)$ with their orbits on the projective line (Lemmas 7-15), we have to consider the following subcases:

(1.3) (i) $P S L(2, q)_{B}$ is conjugate to $S_{4}$, and $k=6$ or 8 .

(1.3) (ii) $\operatorname{PSL}(2, q)_{B}$ is conjugate to $A_{5}$, and $k=6,10,12$ or 20.

(1.3) (iii) $\operatorname{PSL}(2, q)_{B}$ is conjugate to a semi-direct product of an elementary Abelian subgroup of order $\bar{q} \mid q$ with a cyclic subgroup of order $c$ of $P S L(2, q)$ with $c \mid \bar{q}-1$ and $c \mid q-1$, and $k=\bar{q}$.

(1.3) (iv) $P S L(2, q)_{B}$ is conjugate to $P S L(2, \bar{q})$ with $\bar{q}^{m}=q, m \geq 1$, and $k=\bar{q}+1$ or $\bar{q}(\bar{q}-1)$ if $m$ is even.

(1.3) (v) $\operatorname{PSL}(2, q)_{B}$ is conjugate to $P G L(2, \bar{q})$ with $\bar{q}^{m}=q, m>1$ even, and $k=$ $\bar{q}+1$ or $\bar{q}(\bar{q}-1)$.

(1.3)(i): We may assume that $q$ is odd. Applying Eqs. (1) and (4) yields for $k=6$ that $q$ is not a prime power, and for $k=8$ that $q=37$, in which case $q \equiv \pm 1(\bmod$ 8) (cf. Lemma 14) does not hold.

(1.3)(ii): Again, we may assume that $q$ is odd and consider Eqs. (1) and (4) for the given values of $k$. We obtain for $k=6$ that $q=5$, which is clearly impossible due to Corollary 5, for $k=10$ that $q$ is not a prime power, and for $k=20$ that $q=971$, in which case Lemma 3(c) gives a contradiction. If $k=12$, then we get $q=101$. Since $\left|P S L(2, q)_{0 B}\right|=5$ by Eq. (4) and $5 \mid \frac{q-1}{2}, P S L(2, q)_{0 B}$ has two distinct fixed points. If one fixed point lies outside $B$, then clearly $q \equiv 1(\bmod 5)$ and hence $k=12$ is not possible. Thus, we may assume that both fixed points are incident with $B$. But then, as every non-identity element of $\operatorname{PSL}(2, q)$ fixes at most two distinct points, $P S L(2, q)_{0 B}$ must fix some 2-subset by the definition of Steiner 4-designs, and hence contains an involution, a contradiction.

(1.3)(iii): We have $\left((q-2)\left|P S L(2, q)_{0 B}\right| \cdot n+6, q\right)=\left(2 \cdot\left|P S L(2, q)_{0 B}\right| \cdot n-\right.$ $6, q)$, and property (3) gives in particular

$$
k|2 \cdot| P S L(2, q)_{0 B} \mid \cdot n-6 .
$$


On the other hand, it follows from Eq. (4) that $\left|P S L(2, q)_{0 B}\right| \mid k-1$. Therefore, we have in particular

$$
\frac{k-1}{2 n}<\frac{k+6}{2 n} \leq\left|P S L(2, q)_{0 B}\right| \mid k-1 .
$$

a) If $q$ is even, then we deduce that $\left|P S L(2, q)_{0 B}\right|=k-1$. Property (5) yields that $k \mid 2 k-8$, and, as clearly $(2 k-8, k)=(8, k)$, only $k=8$ is possible. Thus, we have $q=32$ in view of Eq. (1), which is impossible by Lemma 3 (c).

b) If $q$ is odd, then by property (6), we have to consider the possibilities when $\left|P S L(2, q)_{0 B}\right|=\frac{k-1}{\bar{n}}$ with $\bar{n}=1,2$, 3. If $\left|P S L(2, q)_{0 B}\right|=k-1$, then we obtain $k \mid 4 k-10$ by property (3). Clearly, $(4 k-10, k)=(10, k)$, but as $k \mid q$, only $k=5$ is possible. Then, Eq. (1) gives $q=5$, which leads to a contradiction in view of Corollary 5. For $\left|P S L(2, q)_{0 B}\right|=\frac{k-1}{\overline{2}}$, we have $k \mid 2 k-8$ and thus $k=8$ as above, which is impossible as $k \nmid q$. If $\left|P S L(2, q)_{0 B}\right|=\frac{k-1}{\overline{3}}$, then property (3) yields $k \mid \frac{4 k-22}{3}$. Since $(4 k-22,3 k)=(22,3 k)$ is not divisible by 3 , this is not possible.

(1.3)(iv): We have $\left|P S L(2, q)_{0 B}\right|=\frac{\bar{q}(\bar{q}-1)}{n}$ if $k=\bar{q}+1$. Thus, Eq. (1) yields for $k=\bar{q}+1$ that $q=\bar{q}$ must hold, which is impossible due to Corollary 5. For $m>1$ even and $k=\bar{q}(\bar{q}-1)$, it follows that $\left|P S L(2, q)_{0 B}\right|=\frac{\bar{q}+1}{n}$. Hence, property (3) gives

$$
\bar{q}(\bar{q}-1) \mid(q-2)(\bar{q}+1)+6=\bar{q}^{m+1}+\bar{q}^{m}-2 \bar{q}+4 .
$$

Since $\left(\bar{q}^{m+1}+\bar{q}^{m}-2 \bar{q}+4, \bar{q}\right)=(4, \bar{q})$ and $k>4$, only the case when $\bar{q}=4$ has to be considered. Thus, $k=12$ and applying Eq. (2) immediately gives a contradiction.

(1.3)(v): Clearly $n$ does not appear in Eqs. (1) and (2) as well as in property (3), and we may argue mutatis mutandis as in subcase (iv).

Now, let us assume that $N<G \leq \operatorname{Aut}(N)$. We recall that $q=p^{e}>3$, and will distinguish in the following the cases $p>2$ and $p=2$.

First, let $p>2$. We define $G^{*}=G \cap\left(P S L(2, q) \rtimes\left\langle\tau_{\alpha}\right\rangle\right)$ with $\tau_{\alpha} \in$ $\operatorname{Sym}\left(G F\left(p^{e}\right) \cup\{\infty\}\right) \cong S_{v}$ of order $e$ induced by the Frobenius automorphism $\alpha: G F\left(p^{e}\right) \longrightarrow G F\left(p^{e}\right), x \mapsto x^{p}$. Then, by Dedekind's law, we can write

$$
G^{*}=\operatorname{PSL}(2, q) \rtimes\left(G^{*} \cap\left\langle\tau_{\alpha}\right\rangle\right) .
$$

Defining $P \Sigma L(2, q)=P S L(2, q) \rtimes\left\langle\tau_{\alpha}\right\rangle$, it can easily by calculated that $P \Sigma L(2, q)_{0,1, \infty}=\left\langle\tau_{\alpha}\right\rangle$, and $\left\langle\tau_{\alpha}\right\rangle$ has precisely $p+1$ distinct fixed points (cf., e.g., [14, Ch. 6.4, Lemma 2]). As $p>2$, we conclude therefore that $G^{*} \cap\left\langle\tau_{\alpha}\right\rangle \leq G_{0 B}^{*}$ for some appropriate, unique block $B \in \mathcal{B}$ by the definition of Steiner 4-designs. Furthermore, clearly $\operatorname{PSL}(2, q) \cap\left(G^{*} \cap\left\langle\tau_{\alpha}\right\rangle\right)=1$. Thus, if we assume that $G^{*} \leq \operatorname{Aut}(\mathcal{D})$ acts already flag-transitively on $\mathcal{D}$, then we obtain $\left|(0, B)^{G^{*}}\right|=\left|(0, B)^{P S L(2, q)}\right|=b k$ in view of Remark 6. Hence, $\operatorname{PSL}(2, q)$ must also act flag-transitively on $\mathcal{D}$, which has already been considered. Therefore, let us assume that $G^{*} \leq \operatorname{Aut}(\mathcal{D})$ does not act flag-transitively on $\mathcal{D}$. Then $\left|G: G^{*}\right|=2$ and $G^{*}$ has exactly two orbits of equal length on the set of flags. It follows for the orbit containing the flag $(0, B)$ that $\left|(0, B)^{G^{*}}\right|=\left|(0, B)^{P S L(2, q)}\right|=\frac{b k}{2}$. As $\operatorname{PSL}(2, q)$ is normal in $G$, we have under $\operatorname{PSL}(2, q)$ also precisely one further orbit of equal length on the set of flags. Then, 
proceeding similarly to the case $N=G$ for each orbit on the set of flags, we obtain (representative for the orbit containing the flag $(0, B)$ ) that

$$
\frac{(q-2)\left|P S L(2, q)_{0 B}\right| \cdot n}{2}=(k-1)(k-2)(k-3)
$$

which is equivalent to

$$
\frac{(q-2)\left|P S L(2, q)_{0 B}\right| \cdot n}{2}+6=k\left(k^{2}-6 k+11\right) .
$$

Hence, we have in particular

$$
k \mid \frac{(q-2)\left|P S L(2, q)_{0 B}\right| \cdot n}{2}+6 .
$$

Since $\operatorname{PSL}(2, q)_{B}$ can have one or two orbits of equal length on the points of $B$, we have

$$
k \text { or } \frac{k}{2}=\left|0^{P S L(2, q)_{B}}\right|=\left|P S L(2, q)_{B}: \operatorname{PSL}(2, q)_{0 B}\right| .
$$

Let us recall that here $q$ is always odd. First considering the case (2.1) when $\left|P S L(2, q)_{0 B}\right|=1$ yields immediately a contradiction to Eq. (8). Let us now observe the case (2.2) when $\left|P S L(2, q)_{0 B}\right|=2$. We have $k \mid 2(q+1)$ in view of property (10), and $k=\left|P S L(2, q)_{B}\right|$ or $\frac{\left|P S L(2, q)_{B}\right|}{2}$ by Eq. (11). a) For $k=\left|P S L(2, q)_{B}\right|$, clearly Eq. (8) with $\left|P S L(2, q)_{0 B}\right|=2$ is equivalent to Eq. (1) with $\left|P S L(2, q)_{0 B}\right|=1$, and thus we can argue exactly as in case (1.1.b). b) For $k=\frac{\left|P S L(2, q)_{B}\right|}{2}$, we have to consider the following subgroups of $P S L(2, q)$ :

(2.2.b) (i) $\operatorname{PSL}(2, q)_{B}$ is conjugate to a cyclic subgroup of order $c$ with $c \mid \frac{q+1}{2}$ of $P S L(2, q)$, and $k=\frac{c}{2}$.

(2.2.b) (ii) $P S L(2, q)_{B}$ is conjugate to a dihedral subgroup of order $2 c$ with $c \mid \frac{q+1}{2}$ of $\operatorname{PSL}(2, q)$, and $k=c$.

(2.2.b) (iii) $P S L(2, q)_{B}$ is conjugate to $A_{4}$, and $k=6$.

(2.2.b) (iv) $P S L(2, q)_{B}$ is conjugate to $S_{4}$, and $k=12$.

(2.2.b) (v) $P S L(2, q)_{B}$ is conjugate to $A_{5}$, and $k=30$.

(2.2.b)(i): Obviously, $k$ cannot take the given value due to Lemma 7.

(2.2.b)(ii): It follows from Eq. (8) that

$$
c \mid \frac{q+1}{2}=\frac{(c-1)(c-2)(c-3)+6}{4}=\frac{c\left(c^{2}-6 c+11\right)}{4} .
$$

As 4 does not divide $c^{2}-6 c+11$, this is impossible.

(2.2.b)(iii)-(v): In view of Eq. (8), we obtain in subcase (iii) that $q=32$ which is not possible, and in each of the other subcases that $q$ is not a prime power. 
We examine finally the case (2.3) when $\left|P S L(2, q)_{0 B}\right|>2$. Combining Eqs. (8) and (11), we obtain

$$
\begin{aligned}
& \frac{(q-2)\left|P S L(2, q)_{B}\right| \cdot n}{2}=k(k-1)(k-2)(k-3) \\
& \text { with } k=\left|0^{P S L(2, q)_{B}}\right|=\frac{\left|P S L(2, q)_{B}\right|}{\left|P S L(2, q)_{0 B}\right|} \text {, or } \\
& (q-2)\left|P S L(2, q)_{B}\right| \cdot n=k(k-1)(k-2)(k-3) \\
& \text { with } k=2 \cdot\left|0^{P S L(2, q)_{B}}\right|=2 \cdot \frac{\left|P S L(2, q)_{B}\right|}{\left|P S L(2, q)_{0 B}\right|} \text {. }
\end{aligned}
$$

In view of the subgroups of $\operatorname{PSL}(2, q)$ with their orbits on the projective line (Lemmas 7-15), we have the following possibilities:

(2.3) (i) $P S L(2, q)_{B}$ is conjugate to $A_{4}$, and $k=2 \cdot\left|0^{P S L(2, q)_{B}}\right|=8$.

(2.3) (ii) $P S L(2, q)_{B}$ is conjugate to $S_{4}$, and $k=\left|0^{P S L(2, q)_{B}}\right|=6$ or 8 , respectively $k=2 \cdot\left|0^{P S L(2, q)_{B}}\right|=8,12$ or 16 .

(2.3) (iii) $\operatorname{PSL}(2, q)_{B}$ is conjugate to $A_{5}$, and $k=\left|0^{P S L(2, q)_{B}}\right|=6,10,12$ or 20 , respectively $k=2 \cdot\left|0^{P S L(2, q)_{B}}\right|=12,20,24$ or 40 .

(2.3) (iv) $P S L(2, q)_{B}$ is conjugate to a semi-direct product of an elementary Abelian subgroup of order $\bar{q} \mid q$ with a cyclic subgroup of order $c$ of $\operatorname{PSL}(2, q)$ with $c \mid \bar{q}-1$ and $c \mid q-1$, and $k=\left|0^{P S L(2, q)_{B}}\right|=\bar{q}$, respectively $k=2$. $\left|0^{P S L(2, q)_{B}}\right|=2 \bar{q}$.

(2.3) (v) $\operatorname{PSL}(2, q)_{B}$ is conjugate to $\operatorname{PSL}(2, \bar{q})$ with $\bar{q}^{m}=q, m \geq 1$, and $k=$ $\left|0^{P S L(2, q)_{B}}\right|=\bar{q}+1$, or $\bar{q}(\bar{q}-1)$ if $m$ is even, respectively $k=2 \cdot\left|0^{P S L(2, q)_{B}}\right|=$ $2(\bar{q}+1)$, or $2 \bar{q}(\bar{q}-1)$ if $m$ is even.

(2.3) (vi) $\operatorname{PSL}(2, q)_{B}$ is conjugate to $\operatorname{PGL}(2, \bar{q})$ with $\bar{q}^{m}=q, m>1$ even, and $k=$ $\left|0^{P S L(2, q)_{B}}\right|=\bar{q}+1$ or $\bar{q}(\bar{q}-1)$, respectively $k=2 \cdot\left|0^{P S L(2, q)_{B}}\right|=2(\bar{q}+1)$ or $2 \bar{q}(\bar{q}-1)$.

(2.3)(i): By Eq. (13), we obtain that $q$ is not a prime power.

(2.3)(ii): First, applying Eq. (12) yields for $k=6$ that $q=17$, which can be excluded since there does not exist any $4-(18,6,1)$ design as already mentioned, and for $k=8$ that $q$ is not a prime power. Using Eq. (13) gives for $k=8$ that $q=37$, in which case $q \equiv \pm 1(\bmod 8)$ (cf. Lemma 14$)$ does not hold, and for $k=12$ and 16 that $q$ is not a prime power in each case.

(2.3)(iii): Observing first Eq. (12) yields for each given value of $k$ that $q$ would be even. Now, applying Eq. (13) gives for $k=12$ the prime $q=101$, which is impossible since according to Lemma 15 we only have orbits of length 6 when $p=5$, and for $k=20$ that $q=971$, in which case Lemma 3 (c) gives a contradiction. For $k=24$ and 40, we obtain in each case that $q$ is not a prime power.

(2.3)(iv): Let $k=\bar{q}$. As $\left(\frac{(q-2)\left|P S L(2, q)_{0 B}\right| \cdot n}{2}+6, q\right)=\left(\left|P S L(2, q)_{0 B}\right| \cdot n-6, q\right)$, property (10) implies that

$$
k|| P S L(2, q)_{0 B} \mid \cdot n-6 .
$$


On the other hand, as $k=\left|0^{P S L(2, q)_{B}}\right|=\left|P S L(2, q)_{B}: P S L(2, q)_{0 B}\right|$, we have $\left|P S L(2, q)_{0 B}\right|=c \mid k-1$. Thus, in particular

$$
\frac{k-1}{n}<\frac{k+6}{n} \leq\left|P S L(2, q)_{0 B}\right| \mid k-1,
$$

and hence $\left|P S L(2, q)_{0 B}\right|=k-1$ as $q$ is odd. But, property (14) gives $k \mid 2 k-8$, and as clearly $(2 k-8, k)=(8, k)$, it would follow that $k=8$, which is impossible since $q$ is odd. For $k=2 \bar{q}$, it follows from Eq. (13) that

$$
(q-2) n=4 \cdot \frac{(\bar{q}-1)}{c}(2 \bar{q}-1)(2 \bar{q}-3),
$$

which gives a contradiction as clearly the left hand side of the equation is not divisible by 4 .

(2.3)(v): For $k=\bar{q}+1$, it follows from Eq. (12) that $q=2(\bar{q}-1)$, which is obviously impossible for $\bar{q}>2$. If $m>1$ even and $k=\bar{q}(\bar{q}-1)$, then we have

$$
(q-2)(\bar{q}+1)=2\left(\bar{q}^{2}-\bar{q}-1\right)\left(\bar{q}^{2}-\bar{q}-2\right)\left(\bar{q}^{2}-\bar{q}-3\right)
$$

in view of Eq. (12), which is impossible since $\bar{q}^{2}-\bar{q}-2=(\bar{q}+1)(\bar{q}-2)$ and $q$ is odd. If $k=2(\bar{q}+1)$, then applying Eq. (13) gives

$$
(q-2)(\bar{q}-1)=4(2 \bar{q}+1)(2 \bar{q}-1) .
$$

Clearly $(4(2 \bar{q}+1)(2 \bar{q}-1), \bar{q}-1)=(\bar{q}-1,12)$, and the few possibilities for $\bar{q}$ can be ruled as $q=\bar{q}^{m}$. For $m>1$ even and $k=2 \bar{q}(\bar{q}-1)$, Eq. (13) yields

$$
(q-2)(\bar{q}+1)=4\left(2 \bar{q}^{2}-2 \bar{q}-1\right)\left(\bar{q}^{2}-\bar{q}-1\right)\left(2 \bar{q}^{2}-2 \bar{q}-3\right) .
$$

Here $\left(4\left(2 \bar{q}^{2}-2 \bar{q}-1\right)\left(\bar{q}^{2}-\bar{q}-1\right)\left(2 \bar{q}^{2}-2 \bar{q}-3\right), \bar{q}+1\right)=(\bar{q}+1,12)$, and the few possibilities for $\bar{q}$ can be ruled out again.

(2.3)(vi): Clearly $n$ does not appear in Eqs. (12) and (13), and we may argue mutatis mutandis as in subcase (v).

Now, let $p=2$. Then clearly $N=P S L(2, q)=P G L(2, q)$, and $\operatorname{Aut}(N)=$ $P \Sigma L(2, q)$. If we assume that $\left\langle\tau_{\alpha}\right\rangle \leq P \Sigma L(2, q)_{0 B}$ for some appropriate, unique block $B \in \mathcal{B}$, then $\operatorname{PSL}(2, q)$ must also be flag-transitive, which has already been considered. Hence, we may assume that $\left\langle\tau_{\alpha}\right\rangle \not \leq P \Sigma L(2, q)_{0 B}$. Let $s$ be a prime divisor of $e=\left|\left\langle\tau_{\alpha}\right\rangle\right|$. As the normal subgroup $H:=\left(P \Sigma L(2, q)_{0,1, \infty}\right)^{s} \leq\left\langle\tau_{\alpha}\right\rangle$ of index $s$ fixes at least four distinct points, we have $G \cap H \leq G_{0 B}$ for some appropriate, unique block $B \in \mathcal{B}$ by the definition of Steiner 4-designs. It can then be deduced that $e=s^{u}$ for some $u \in \mathbb{Z}^{+}$, since if we assume for $G=P \Sigma L(2, q)$ that there exists a further prime divisor $\bar{s}$ of $e$ with $\bar{s} \neq s$ then $\bar{H}:=\left(P \Sigma L(2, q)_{0,1, \infty}\right)^{\bar{s}} \leq\left\langle\tau_{\alpha}\right\rangle$ and $H$ are both subgroups of $P \Sigma L(2, q)_{0 B}$ by the flag-transitivity of $P \Sigma L(2, q)$, and hence $\left\langle\tau_{\alpha}\right\rangle \leq P \Sigma L(2, q)_{0 B}$, a contradiction. Furthermore, as $\left\langle\tau_{\alpha}\right\rangle \not \leq P \Sigma L(2, q)_{0 B}$, we may, $\underline{\text { Springer }}$ 
by applying Dedekind's law, assume that

$$
G_{0 B}=P S L(2, q)_{0 B} \rtimes(G \cap H) .
$$

Clearly, $k=\left|0^{G_{B}}\right|=\left|G_{B}: G_{0 B}\right|$. If $G=P S L(2, q) \rtimes(G \cap H)$, then $\operatorname{PSL}(2, q)$ itself must be flag-transitive. Therefore, we may assume that $G=P \Sigma L(2, q)$. Thus, by Remark 6, we obtain

$$
(q-2)\left|P S L(2, q)_{0 B}\right|=(k-1)(k-2)(k-3) s
$$

$$
\text { with }\left|P S L(2, q)_{0 B}\right|=\frac{\left|P S L(2, q)_{B}\right|}{k} \cdot \begin{cases}s, & \text { if } G_{B}=P S L(2, q)_{B} \rtimes\left\langle\tau_{\alpha}\right\rangle \\ 1, & \text { if } G_{B}=P S L(2, q)_{B} \rtimes H .\end{cases}
$$

Clearly, for each $B \in \mathcal{B}$ there exists always a Klein four-group $V_{4} \leq P S L(2, q)$, which fixes $B$ by the definition of Steiner 4-designs, and some additional point $x \in X$. We will distinguish two cases according as $x$ is incident with $B$ or not and examine for each case the list of possible subgroups of $\operatorname{PSL}(2, q)$ with their orbits on the projective line (cf. Lemmas 7-15). First, let $x \in B$. Then, clearly $k \equiv 1(\bmod 4)$. It follows that we only have to consider the subcase when $\operatorname{PSL}(2, q)_{B}$ is conjugate to $\operatorname{PSL}(2, \bar{q})$ with $\bar{q}^{m}=q, m \geq 1$. In view of Lemma 11 , we obtain $k=\bar{q}+1$. Hence, Eq. (15) gives

$$
(q-2)\left|P S L(2, q)_{0 B}\right|=\bar{q}(\bar{q}-1)(\bar{q}-2) s
$$

with $\left|P S L(2, q)_{0 B}\right|=\bar{q}(\bar{q}-1) \cdot\left\{\begin{array}{l}s, \text { or } \\ 1 .\end{array}\right.$

Since $q=2^{s^{u}}$, we can write $\bar{q}=2^{s^{w}}$ for some integer $0 \leq w \leq u$, and $q=\bar{q}^{m}=\bar{q}^{s^{u-w}}$. As $k=\bar{q}+1=2^{s^{w}}+1>4$, it follows in particular that $w \geq 1$, and thus $s<2^{s^{w}}=$ $\bar{q}$. Therefore, using Eq. (16), we obtain

$$
\bar{q}^{s^{u-w}}-2=q-2 \leq(\bar{q}-2) s<\bar{q}^{2}-2 s .
$$

But, as clearly $u-w \geq 1$ (otherwise $k=q+1$, a contradiction to Corollary 5) this yields a contradiction for every prime $s$.

Now, let $x \notin B$. Then clearly $k \equiv 0(\bmod 4)$. We may restrict ourselves to the examination of the following subcases:

(i) $\operatorname{PSL}(2, q)_{B}$ is conjugate to $A_{4}$ for $s=2$, and $k=12$ in view of Lemma 13.

(ii) $\operatorname{PSL}(2, q)_{B}$ is conjugate to an elementary Abelian subgroup of order $\bar{q} \mid q$ of $P S L(2, q)$, and $k=\bar{q}$ due to Lemma 9.

(iii) $\operatorname{PSL}(2, q)_{B}$ is conjugate to a semi-direct product of an elementary Abelian subgroup of order $\bar{q} \mid q$ with a cyclic subgroup of order $c$ of $P S L(2, q)$ with $c \mid \bar{q}-1$ and $c \mid q-1$, and $k=\bar{q}$ or $\bar{q} c$ by Lemma 10 . 
(iv) $\operatorname{PSL}(2, q)_{B}$ is conjugate to $\operatorname{PSL}(2, \bar{q})$ with $\bar{q}^{m}=q, m \geq 1$, acting outside the $\bar{q}+1$ points mentioned in the case where $x$ has been incident with $B$, and Lemma 11 yields $k=\bar{q}(\bar{q}-1)$ if $m$ is even, or $k=(\bar{q}+1) \bar{q}(\bar{q}-1)$.

Again, we can write in the following $\bar{q}=2^{s^{w}}$ for some integer $0 \leq w \leq u$, and $q=\bar{q}^{m}=\bar{q}^{s^{u-w}}$.

(i): Applying Eq. (15) yields

$$
\begin{aligned}
& (q-2)\left|P S L(2, q)_{0 B}\right|=11 \cdot 10 \cdot 9 \cdot 2 \\
& \text { with }\left|P S L(2, q)_{0 B}\right|=\left\{\begin{array}{l}
2, \text { or } \\
1
\end{array},\right.
\end{aligned}
$$

which is clearly impossible.

(iii): Let $k=\bar{q}$. By Eq. (15), we have

$$
\begin{aligned}
& (q-2)\left|P S L(2, q)_{0 B}\right|=(\bar{q}-1)(\bar{q}-2)(\bar{q}-3) s \\
& \text { with }\left|P S L(2, q)_{0 B}\right|=c \cdot\left\{\begin{array}{l}
s, \text { or } \\
1 .
\end{array}\right.
\end{aligned}
$$

As $k=\bar{q}=2^{s^{w}}>4$, we have in particular $w \geq 1$, and hence $s<2^{s^{w}}=\bar{q}$. Thus, using Eq. (17), we obtain

$$
q-2=\bar{q}^{s^{u-w}}-2<\bar{q}^{3} s<\bar{q}^{4} .
$$

Since clearly $u-w \geq 1$ (otherwise $k=q$, which is not possible by Corollary 5) this yields a contradiction for $s \geq 5$. If $s=2$, then $\bar{q}^{2^{u-w}}-2<2 \bar{q}^{3}$ must hold, which cannot be true for $u-w>1$. For $s=3$, we may also assume that $u-w=1$ since otherwise we would have $q=\bar{q}^{3^{u-w}} \geq \bar{q}^{9}$, again a contradiction to the inequality above. As $c \mid \bar{q}-1$, it follows for both cases from Eq. (17) that

$$
\bar{q}-2 \mid q-2
$$

and hence

$$
2^{s^{w}-1}-1 \mid 2^{s^{u}-1}-1
$$

Thus, clearly

$$
s^{w}-1 \mid s^{u}-1
$$

and

$$
w \mid u \text {. }
$$

Therefore, we may conclude that $w=1$ and $u=2$. For $s=2$, it follows that $k=\bar{q}=$ 4, which has been excluded. For $s=3$, we have $\bar{q}=8$ and $q=512$, and Eq. (17) $\underline{\text { Springer }}$ 
yields

$$
\begin{aligned}
& 510 \cdot\left|\operatorname{PSL}(2, q)_{0 B}\right|=7 \cdot 6 \cdot 5 \cdot 3 \\
& \text { with }\left|\operatorname{PSL}(2, q)_{0 B}\right|=c \cdot\left\{\begin{array}{l}
3, \text { or } \\
1
\end{array},\right.
\end{aligned}
$$

which is clearly impossible.

Now, let $k=\bar{q} c$. Then Eq. (15) yields

$$
\begin{aligned}
(q-2)\left|P S L(2, q)_{0 B}\right| & =(\bar{q} c-1)(\bar{q} c-2)(\bar{q} c-3) s \\
\text { with }\left|P S L(2, q)_{0 B}\right| & =\left\{\begin{array}{l}
s, \text { or } \\
1 .
\end{array}\right.
\end{aligned}
$$

Polynomial division with remainder gives

$$
2^{s^{u}-1}-1=\left(\sum_{i=1}^{\bar{m}} \frac{2^{s^{u}-1}}{\left(c \cdot 2^{s^{w}-1}\right)^{i}}\right)\left(c \cdot 2^{s^{w}-1}-1\right)+\frac{2^{s^{u}-1}}{\left(c \cdot 2^{s^{w}-1}\right)^{\bar{m}}}-1
$$

for a suitable $\bar{m} \in \mathbb{Z}^{+}$(such that

$$
\operatorname{deg}\left(\frac{2^{s^{u}-1}}{\left(c \cdot 2^{s^{w}-1}\right)^{\bar{m}}}-1\right)<\operatorname{deg}\left(c \cdot 2^{s^{w}-1}-1\right)
$$

as is well-known). As $c$ is odd, clearly $\left(\frac{2^{s^{u}-1}}{c \cdot 2^{w^{u}}-1}\right)^{\bar{m}} \neq 1$, and it follows that $\bar{q} c-2$ does not divide $q-2$, yielding a contradiction to Eq. (18).

(ii): Let $k=\bar{q}$. By Eq. (15), we have

$$
\begin{aligned}
(q-2)\left|P S L(2, q)_{0 B}\right| & =(\bar{q}-1)(\bar{q}-2)(\bar{q}-3) s \\
\text { with }\left|P S L(2, q)_{0 B}\right| & =\left\{\begin{array}{l}
s, \text { or } \\
1 .
\end{array}\right.
\end{aligned}
$$

As it is easily seen we may argue mutatis mutandis as in subcase (iii), $k=\bar{q}$.

(iv): If $m>1$ even and $k=\bar{q}(\bar{q}-1)$, then in view of Eq. (15), we have

$$
\begin{aligned}
(q-2)\left|P S L(2, q)_{0 B}\right| & =\left(\bar{q}^{2}-\bar{q}-1\right)\left(\bar{q}^{2}-\bar{q}-2\right)\left(\bar{q}^{2}-\bar{q}-3\right) s \\
\text { with }\left|P S L(2, q)_{0 B}\right| & =(\bar{q}+1) \cdot\left\{\begin{array}{l}
s, \text { or } \\
1 .
\end{array}\right.
\end{aligned}
$$

As obviously $\bar{q}^{2}-\bar{q}-2=(\bar{q}+1)(\bar{q}-2)$, this leads to a contradiction analogously as in subcase (iii), $k=\bar{q}$. For $k=\bar{q}^{3}-\bar{q}$, Eq. (15) yields

$$
\begin{aligned}
(q-2)\left|P S L(2, q)_{0 B}\right| & =\left(\bar{q}^{3}-\bar{q}-1\right)\left(\bar{q}^{3}-\bar{q}-2\right)\left(\bar{q}^{3}-\bar{q}-3\right) s \\
\text { with }\left|P S L(2, q)_{0 B}\right| & =\left\{\begin{array}{l}
s, \text { or } \\
1 .
\end{array}\right.
\end{aligned}
$$


We already know that $k=(\bar{q}+1) \bar{q}(\bar{q}-1) \equiv 0(\bmod 4)$, and thus $\bar{q}>2$. If $\left|P S L(2, q)_{0 B}\right|=s$, then

$$
\begin{aligned}
q & =\left(\bar{q}^{3}-\bar{q}-1\right)\left(\bar{q}^{3}-\bar{q}-2\right)\left(\bar{q}^{3}-\bar{q}-3\right)+2=\bar{q}^{9}-l \\
\text { with } l & =3 \bar{q}^{7}+6 \bar{q}^{6}-3 \bar{q}^{5}-12 \bar{q}^{4}-10 \bar{q}^{3}+6 \bar{q}^{2}+11 \bar{q}+4 .
\end{aligned}
$$

As clearly $l>0$, we have $q<\bar{q}^{9}$. On the other hand, for $\bar{q}>2$ certainly $l<\bar{q}^{8}(\bar{q}-1)$ and hence $q>\bar{q}^{8}$ must hold, which is impossible since $q=\bar{q}^{m}$. If $\left|P \operatorname{SL}(2, q)_{0 B}\right|=1$, then Eq. (19) yields

$$
\begin{aligned}
q=l s+2 \text { with } l & =\left(\bar{q}^{3}-\bar{q}-1\right)\left(\bar{q}^{3}-\bar{q}-2\right)\left(\bar{q}^{3}-\bar{q}-3\right) \\
& =\bar{q}^{9}-3 \bar{q}^{7}-6 \bar{q}^{6}+3 \bar{q}^{5}+12 \bar{q}^{4}+10 \bar{q}^{3}-6 \bar{q}^{2}-11 \bar{q}-6
\end{aligned}
$$

Since $\bar{q}=2^{s^{w}}>2$, we conclude that $w \geq 1$ and $s<2^{s^{w}}=\bar{q}$. As obviously $l<\bar{q}^{9}-1$, it follows that $q<\left(\bar{q}^{9}-1\right) \bar{q}+2<\bar{q}^{10}$. On the other hand, for $\bar{q}>2$ clearly $q=l s+2 \geq 2(l+1)>\bar{q}^{9}$ must hold, which again is impossible.

Subcase $N=\operatorname{PSL}(d, q), d \geq 3$.

We have here $\operatorname{Aut}(N)=P \Gamma L(d, q) \rtimes\left\langle\iota_{\beta}\right\rangle$, where $\iota_{\beta}$ denotes the graph automorphism induced by the inverse-transpose map $\beta: G L(d, q) \longrightarrow G L(d, q), x \mapsto{ }^{t}\left(x^{-1}\right)$. In the following, let $n=(d, q-1)$.

Let us first assume that $d=3$. In order to show that $G$ with $\operatorname{PSL}(3, q)$ as simple normal subgroup cannot act on any non-trivial $4-\left(q^{2}+q+1, k, 1\right)$ design, we prove first that $k \leq q+1$. It is well-known that, for any line $\mathcal{G}$ in the underlying projective plane $P G(2, q)$, the translation group $T(\mathcal{G})$ operates regularly on the points of $P G(2, q) \backslash \mathcal{G}$ and acts trivially on $\mathcal{G}$. Thus $T(\mathcal{G})$ fixes a block $B \in \mathcal{B}$ if four or more distinct points of $B$ lie on $\mathcal{G}$. By the definition of Steiner 4-designs, we may choose in $P G(2, q)$ four distinct collinear points $x_{1}, x_{2}, x_{3}, x_{4} \in X$, which are incident with a unique block $B \in \mathcal{B}$. Let $\mathcal{G}$ denote the line of $P G(2, q)$ through $x_{1}, x_{2}, x_{3}, x_{4} \in X$. Consequently, if the block $B$ contains at least one further point of $P G(2, q) \backslash \mathcal{G}$, then it must contain all points of $P G(2, q) \backslash \mathcal{G}$, thus at least $q^{2}+4$ many, which is not possible as $k \leq\left\lfloor\frac{v}{5}+3\right\rfloor$ by Proposition 4 (a). Therefore, $B$ is completely contained in $\mathcal{G}$, and hence $k \leq q+1$.

Now, by the definition of Steiner 4-designs, we may consider a 4-subset consisting of three distinct collinear points $x_{1}, x_{2}, x_{3} \in X$ and one non-collinear point $x_{4} \in X$, which is incident with a unique block $B \in \mathcal{B}$. If $B$ contains a fourth point on the line $\mathcal{G}$ of $P G(2, q)$ through $x_{1}, x_{2}, x_{3} \in X$, then by the same arguments as above using the translation group $T(\mathcal{G})$, we conclude that $B$ lies completely in $\mathcal{G}$, a contradiction. Thus, we may assume that $B$ contains only further points which are not on $\mathcal{G}$. Without restriction, we may identify $x_{1}=\langle(1,0,0)\rangle, x_{2}=\langle(0,0,1)\rangle, x_{3} \in\left\langle x_{1}, x_{2}\right\rangle$, and $x_{4}=$ $\langle(0,1,0)\rangle$. As it is known the cyclic group

$$
\left\{\left(\begin{array}{lll}
c & & \\
& c^{-2} & \\
& & c
\end{array}\right) \mid c \in G F(q)^{*}\right\}
$$


of linear transformations on the associated vector space $V=V(3, q)$ induces a group $U$ of dilatations of order $\frac{q-1}{n}$ on $P G(2, q)$ with axis the line $\mathcal{G}=\left\langle x_{1}, x_{2}\right\rangle$ and as center the point $x_{4}$. It is clear that $U$ fixes each point of its axis as well as its center. Furthermore, $U$ acts semi-regularly on the points of $P G(2, q) \backslash\left(\mathcal{G} \cup\left\{x_{4}\right\}\right)$ and hence all point-orbits on $P G(2, q) \backslash\left(\mathcal{G} \cup\left\{x_{4}\right\}\right)$ have length $\frac{q-1}{n}$. As $U$ fixes each of the points $x_{1}, x_{2}, x_{3}, x_{4} \in X$ and hence in particular $B$, we get

$$
k \equiv 4\left(\bmod \frac{q-1}{n}\right)
$$

Due to the fact that $k \leq q+1$, this is obviously impossible if $3 \nmid q-1$, and for $3 \mid q-1$, we conclude that

$$
k=\frac{q-1}{3}+4 \quad \text { or } \quad k=2 \cdot \frac{q-1}{3}+4 .
$$

If we assume that $q>7$, then indeed $q \geq 13$ and we obtain $\frac{q-1}{3} \geq 4$, which means that we have at least four distinct collinear points on some line $\mathcal{H}$ of $P G(2, q)$, and we may argue as above using the translation group $T(\mathcal{H})$ that $B$ lies completely in $\mathcal{H}$, which is clearly impossible. Therefore, we only have to consider the cases when $q=4$ or 7 . For $q=7$, condition (20) yields $k=6$ or 8 , whereas $k=6$ can immediately be ruled out using Lemma 3 (d). If any 4-(57, 8, 1) design exists, then there must also exist a derived $3-(56,7,1)$ design. But, for $t=3$, it follows from Lemma 3 (c) that in particular 54 must be divisible by 5 , a contradiction. Now, let us assume that $q=4$. Then only $k=5$ can occur. We have the situation of two intersecting lines $\mathcal{G}$ and $\mathcal{H}$, and we may distinguish the two cases according as their intersecting point $x \in \mathcal{G} \cap \mathcal{H}$ is incident with $B$ or not. In the first case, $\mathcal{G}$ and $\mathcal{H}$ are precisely the lines which intersects $B$ in exactly three distinct points and it can easily be shown that then $\left|P S L(3,4)_{B}\right| \leq 8$. In the second case there is exactly one line which intersects $B$ in exactly three distinct points and it can be verified here that $\left|P S L(3,4)_{B}\right| \leq 2$. However, as there are as blocks 21 projective lines in $P G(2,4)$, it follows that $\left|P S L(3,4)_{B}\right| \geq \frac{|P S L(3,4)|}{b-21}=\frac{20160}{1176}>17$, a contradiction in both cases. Thus $\operatorname{PSL}(3, q)$, and hence also $G$ with $\operatorname{PSL}(3, q)$ as simple normal subgroup, cannot act on any non-trivial $4-\left(q^{2}+q+1, k, 1\right)$ design.

Now, we consider the case when $d>3$. Via induction over $d$, we verify that $G \leq \operatorname{Aut}(\mathcal{D})$ cannot act on any non-trivial Steiner 4-design $\mathcal{D}$. For this, let us assume that there is a counter-example with $d$ minimal. Without restriction, we can choose four distinct points $x_{1}, x_{2}, x_{3}, x_{4}$ from a hyperplane $\mathcal{H}$ of $P G(d-1, q)$. Analogously as above, it can be shown that the unique block $B \in \mathcal{B}$ which is incident with the 4 -subset $\left\{x_{1}, x_{2}, x_{3}, x_{4}\right\}$ is contained completely in $\mathcal{H}$. Thus, $\mathcal{H}$ induces a 4- $\left(\frac{q^{d-1}-1}{q-1}, k, 1\right)$ design, on which $G$ containing $\operatorname{PSL}(d-1, q)$ as simple normal subgroup operates. Inductively, we obtain the minimal counter-example for $d=3$, a contradiction as above. 
Case $N=\operatorname{PSU}\left(3, q^{2}\right), v=q^{3}+1, q=p^{e}>2$.

Here $\operatorname{Aut}(N)=P \Gamma U\left(3, q^{2}\right)$, and $|G|=\left(q^{3}+1\right) q^{3} \frac{\left(q^{2}-1\right)}{n} a$ with $n=(3, q+1)$ and $a \mid 2 n e$. For the existence of flag-transitive Steiner 4-designs, necessarily

$$
r=\frac{q^{3}\left(q^{3}-1\right)\left(q^{3}-2\right)}{(k-1)(k-2)(k-3)}|| G_{0}||\left|P \Gamma U\left(3, q^{2}\right)_{0}\right|=q^{3}\left(q^{2}-1\right) 2 e
$$

must hold in view of Lemma 2 . As obviously $\left(q^{2}+q+1, q+1\right)=1$ and $\left(q^{3}-2\right.$, $q+1)=(3, q+1)=n$, we have in particular

$$
\left(q^{3}-2\right)\left(q^{2}+q+1\right) \mid(k-1)(k-2)(k-3) 2 n e, \text { where } e \leq \log _{2} q .
$$

On the other hand, Corollary 5 yields $k \leq\left\lfloor\sqrt{q^{3}+1}+\frac{5}{2}\right\rfloor<q^{\frac{3}{2}}+3$. Hence, using property (21), we have only a small number of possibilities to check, which can easily be eliminated by hand.

Case $N=S z(q), v=q^{2}+1, q=2^{2 e+1}>2$.

We have $\operatorname{Aut}(N)=S z(q) \rtimes\langle\alpha\rangle$, where $\alpha$ denotes the Frobenius automorphism $G F(q) \longrightarrow G F(q), x \mapsto x^{2}$. Thus, by Dedekind's law, $G=S z(q) \rtimes(G \cap\langle\alpha\rangle)$, and $|G|=\left(q^{2}+1\right) q^{2}(q-1) a$ with $a \mid 2 e+1$. From Remark 6, we obtain

$$
\left(q^{2}-2\right)(q+1)=(k-1)(k-2)(k-3) \frac{a}{\left|G_{x B}\right|} \text { if } x \in B .
$$

First, we show that every element $g \in G$ that fixes three distinct points must fix at least five distinct points. Let us assume that $g \in G$ with $\left|\operatorname{Fix}_{X}(g)\right| \geq 3$. Let $x \in$ $\operatorname{Fix}_{X}(g)$, and $P$ the normal Sylow 2-subgroup of $S z(q)_{x}$ acting regularly on $X \backslash\{x\}$. Set $C:=C_{P}(g)$. If $y, z \in \operatorname{Fix}_{X}(g) \backslash\{x\}$, then $z=y^{h}$ with $h \in P$. Thus, as $y^{h g}=$ $y^{h}=y^{g h}$, we conclude that

$$
\left[h^{-1}, g^{-1}\right] \in G_{x y} \cap\left[P, G_{x}\right] \leq P_{y}=1 .
$$

Then $h \in C$, and hence $C$ acts point-transitively on $\operatorname{Fix}_{X}(g) \backslash\{x\}$. Therefore, as $\left|\operatorname{Fix}_{X}(g)\right| \geq 3$, it follows that $\left|\operatorname{Fix}_{X}(g)\right| \equiv 1(\bmod 2)$. Clearly, the set $\operatorname{Fix}_{X}(g)$ is left invariant by $C_{S_{z}(q)}(g)$ and $C_{S z(q)}(g)$ operates on $\operatorname{Fix}_{X}(g)$. Since $x \in \operatorname{Fix}_{X}(g)$ can be chosen arbitrarily, it follows that $C_{S z(q)}(g)$ operates point-transitively on $\operatorname{Fix}_{X}(g)$, and thus $\left|\operatorname{Fix}_{X}(g)\right||| S z(q) \mid$. As the order of $S z(q)$ is not divisible by 3 , clearly $\mid$ Fix $_{X}(g) \mid \neq 3$, and due to the fact that $\left|\operatorname{Fix}_{X}(g)\right| \equiv 1(\bmod 2)$, we have $\left|\operatorname{Fix}_{X}(g)\right| \geq 5$.

Since $G$ is block-transitive, it is sufficient to consider some appropriate, unique block $B \in \mathcal{B}$. As clearly $\langle\alpha\rangle \leq \operatorname{Aut}(N)_{0,1, \infty}$, it follows from above that $\langle\alpha\rangle$ must fix some fourth point, and hence $G \cap\langle\alpha\rangle \leq G_{0 B}$ by the definition of Steiner 4-designs. Thus, we have particularly

$$
\left(q^{2}-2\right)(q+1) \leq(k-1)(k-2)(k-3),
$$

which does obviously not hold for $k \leq q+2$. On the other hand, Corollary 5 yields $k \leq\left\lfloor\sqrt{q^{2}+1}+\frac{5}{2}\right\rfloor<q+3$, a contradiction. 
Case $N=\operatorname{Re}(q), v=q^{3}+1, q=3^{2 e+1}>3$.

Here $\operatorname{Aut}(N)=\operatorname{Re}(q) \rtimes\langle\alpha\rangle$, where $\alpha$ denotes the Frobenius automorphism $G F(q) \longrightarrow G F(q), x \mapsto x^{3}$. Thus, by Dedekind's law, $G=\operatorname{Re}(q) \rtimes(G \cap\langle\alpha\rangle)$, and $|G|=\left(q^{3}+1\right) q^{3}(q-1) a$ with $a \mid 2 e+1$. It follows from Remark 6 that

$$
\left(q^{3}-2\right)\left(q^{2}+q+1\right)=(k-1)(k-2)(k-3) \frac{a}{\left|G_{x B}\right|} \text { if } x \in B .
$$

First, we show that every element $g \in G$ that fixes three distinct points must also fix a fourth point. Let us assume that $g \in G$ with $\left|\operatorname{Fix}_{X}(g)\right| \geq 3$. Let $x \in \operatorname{Fix}_{X}(g)$, and $P$ the normal Sylow 3-subgroup of $\operatorname{Re}(q)_{x}$ acting regularly on $X \backslash\{x\}$. As in the previous Case, it can be shown that then $C_{P}(g)$ acts point-transitively on $\operatorname{Fix}_{X}(g) \backslash\{x\}$. Thus, we have $\left|\operatorname{Fix}_{X}(g)\right| \equiv 0(\bmod 2)$, and the claim follows.

Since $G$ is block-transitive, it is sufficient to consider some appropriate, unique block $B \in \mathcal{B}$. As clearly $\langle\alpha\rangle \leq \operatorname{Aut}(N)_{0,1, \infty}$, we deduce from above that $G \cap\langle\alpha\rangle \leq$ $G_{0 B}$ by the definition of Steiner 4-designs. Hence, we have in particular

$$
\left(q^{3}-2\right)\left(q^{2}+q+1\right) \leq(k-1)(k-2)(k-3),
$$

which is not possible as Corollary 5 yields $k \leq\left\lfloor\sqrt{q^{3}+1}+\frac{5}{2}\right\rfloor<q^{\frac{3}{2}}+3$.

Case $N=\operatorname{Sp}(2 d, 2), d \geq 3, v=2^{2 d-1} \pm 2^{d-1}$.

As here $|\operatorname{Out}(N)|=1$, we have $N=G$. Let $X^{+}$respectively $X^{-}$denote the set of points on which $G$ operates. It is well-known that $G_{z}$ acts on $X^{ \pm} \backslash\{z\}$ as $O^{ \pm}(2 d, 2)$ does in its usual rank 3 manner on singular points of the underlying non-degenerate orthogonal space $V^{ \pm}=V^{ \pm}(2 d, 2)$.

It is easily seen that there are $2^{2 d-2}\left(2^{d} \mp 1\right)\left(2^{d-1} \pm 1\right)$ hyperbolic pairs in $V^{ \pm}$, and by Witt's theorem, $O^{ \pm}(2 d, 2)$ is transitive on these hyperbolic pairs. Let $\{x, y\}$ denote a hyperbolic pair, and $\mathcal{E}=\langle x, y\rangle$ the hyperbolic plane spanned by $\{x, y\}$. As $\mathcal{E}$ is non-degenerate, we have the orthogonal decomposition

$$
V^{ \pm}=\mathcal{E} \perp \mathcal{E}^{\perp}
$$

Clearly, $O^{ \pm}(2 d, 2)_{\{x, y\}}$ stabilizes $\mathcal{E}^{\perp}$ as a subspace, which implies that $O^{ \pm}(2 d, 2)_{\{x, y\}} \cong O^{ \pm}(2 d-2,2)$. Therefore, we have

$$
O^{ \pm}(2 d-2,2) \cong O^{ \pm}(2 d, 2)_{\{x, y\}} \unlhd O^{ \pm}(2 d, 2)_{\mathcal{E}}=G_{z, \mathcal{E}}
$$

Since $O^{ \pm}(2 d-2,2)$ acts transitively on the singular points of the $(2 d-2)$ dimensional orthogonal subspace, the smallest orbit on $V^{ \pm} \backslash \mathcal{E}$ under $G_{z, \mathcal{E}}$ has length at least $2^{2 d-3} \pm 2^{d-2}$. If the unique block $B \in \mathcal{B}$ which is incident with the 4-subset $\{x, y, x+y, z\}$ contains some singular point in $V^{ \pm} \backslash \mathcal{E}$, then we would have $k \geq 2^{2 d-3} \pm 2^{d-2}+4$, a contradiction to Corollary 5. Thus, all points of $B$ apart from $z$ lie completely in $\mathcal{E}$. By the flag-transitivity of $G$, it follows that for each block all points apart from a singleton must be contained in an affine plane. Thus $k=5$, which is impossible since $k \equiv 0(\bmod 4)$ by Lemma $3(\mathrm{c})$. 
Cases $N=P S L(2,11), v=11$, and $N=M_{11}, v=12$.

As is known, these exceptional permutation actions occur inside the Mathieu group $M_{24}$ in its action on 24 points. This set can be partitioned into two sets $X_{1}$ and $X_{2}$ of 12 points each such that the setwise stabilizer of $X_{1}$ is the Mathieu group $M_{12}$. The stabilizer in this latter group of a point $x$ in $X_{1}$ is isomorphic to $M_{11}$ and operates (apart from its natural 4-transitive action on $\left.X_{1} \backslash\{x\}\right)$ 3-transitively on the 12 points of $X_{2}$. The one-point stabilizer in this action of degree 12 is $\operatorname{PSL}(2,11)$ acting 2-transitively on 11 points. The geometry preserved by the 3-transitive action of $M_{11}$ is not a Steiner $t$-design, but a 3-(12, 6, 2) design (e.g. [2, Ch. IV, 5.3]). Hence also the derived design $\mathcal{D}$ on which $G \leq \operatorname{Aut}(\mathcal{D})$ acts cannot be a Steiner design.

Case $N=M_{v}, v=11,12,22,23,24$.

If $v=11,12,23$ or 24 , then $G=M_{v}$ is always 4-transitive and thus [26, Thm. 3] yields the designs described in the Main Theorem. Obviously, flag-transitivity holds as the 4-transitivity of $G$ implies that $G_{x}$ acts block-transitively on the derived Steiner 3 -design $\mathcal{D}_{x}$ for any $x \in X$. For $v=22$, Corollary 5 gives $k \leq 7$, and again the cases for $k$ can easily be eliminated in view of Lemma 3 (c) and (d).

This completes the proof of the Main Theorem.

Acknowledgements I am grateful to C. Hering for helpful conversations. The author gratefully acknowledges partial support by the Deutsche Forschungsgemeinschaft (DFG).

\section{References}

1. M. Aschbacher, "Chevalley groups of type $G_{2}$ as the group of a trilinear form," J. Algebra 109 (1987), 193-259.

2. Th. Beth, D. Jungnickel, and H. Lenz, Design Theory, Vol. I and II, Encyclopedia of Math. and Its Applications 69/78, Cambridge Univ. Press, Cambridge, 1999.

3. R.E. Block, "Transitive groups of collineations on certain designs," Pacific J. Math. 15 (1965), 13-18.

4. F. Buekenhout, A. Delandtsheer, J. Doyen, P.B. Kleidman, M.W. Liebeck, and J. Saxl, "Linear spaces with flag-transitive automorphism groups," Geom. Dedicata 36 (1990), 89-94.

5. F. Buekenhout, A. Delandtsheer, and J. Doyen, "Finite linear spaces with flag-transitive groups," J. Combin. Theory, Series A 49 (1988), 268-293.

6. F. Buekenhout, "Remarques sur 1'homogénéité des espaces linéaires et des systèmes de blocs," Math. Z. 104 (1968), 144-146.

7. P.J. Cameron and W.M. Kantor, "2-transitive and antiflag transitive collineation groups of finite projective and polar spaces," J. Algebra 60 (1979), 384-422.

8. P.J. Cameron, "Parallelisms of Complete Designs," London Math. Soc. Lecture Note Series 23, Cambridge Univ. Press, Cambridge, 1976.

9. J.H. Conway, R.T. Curtis, S.P. Norton, R.A. Parker, and R.A. Wilson, Atlas of Finite Groups, Clarendon Press, Oxford, 1985.

10. C.W. Curtis, W.M. Kantor, and G.M. Seitz, "The 2-transitive permutation representations of the finite Chevalley groups," Trans. Amer. Math. Soc. 218 (1976), 1-59.

11. A. Delandtsheer, "Finite (line, plane)-flag-transitive planar spaces," Geom. Dedicata 41 (1992), 145153 .

12. A. Delandtsheer, "Dimensional linear spaces," in F. Buekenhout (Ed.), Handbook of Incidence Geometry, Elsevier Science, Amsterdam (1995), pp. 193-294.

13. A. Delandtsheer, "Finite flag-transitive linear spaces with alternating socle," in A. Betten et al. (Eds.), Algebraic Combinatorics and Applications, Proc. Euroconf. (Gößweinstein 1999), Springer, Berlin (2001), pp. 79-88. 
14. P. Dembowski, Finite Geometries, Springer, Berlin, Heidelberg, New York, 1968; Reprint: Springer, 1997.

15. L.E. Dickson, Linear Groups with an Exposition of the Galois Field Theory, Teubner, Leipzig, 1901; Reprint: Dover Publications, New York, 1958.

16. D. Gorenstein, Finite Simple Groups. An Introduction to Their Classification, Plenum Publishing Corp., New York, London, 1982.

17. C. Hering, "Transitive linear groups and linear groups which contain irreducible subgroups of prime order," Geom. Dedicata 2 (1974), 425-460.

18. C. Hering, "Transitive linear groups and linear groups which contain irreducible subgroups of prime order, II," J. Algebra 93 (1985), 151-164.

19. D.G. Higman and J.E. McLaughlin, "Geometric AB A-groups," Illinois J. Math. 5 (1961), 382-397.

20. M. Huber, "Classification of flag-transitive Steiner quadruple systems," J. Combin. Theory, Series A 94 (2001), 180-190.

21. M. Huber, "The classification of flag-transitive Steiner 3-designs," Adv. Geom. 5 (2005), 195-221.

22. M. Huber, On Highly Symmetric Combinatorial Designs, Habilitationsschrift, Univ. Tübingen, Tübingen, 2005, Shaker, Aachen, 2006.

23. B. Huppert, "Zweifach transitive, auflösbare Permutationsgruppen," Math. Z. 68 (1957), 126-150.

24. B. Huppert, Endliche Gruppen I, Springer, Berlin, Heidelberg, New York, 1967.

25. W.M. Kantor, "Flag-transitive planes," in C.A. Baker and L.M. Batten (Eds.), Finite Geometries (Winnipeg, Can., 1984), Lecture Notes in Pure and Applied Math., vol. 103, Dekker, New York (1985), pp. $179-181$.

26. W.M. Kantor, "Homogeneous designs and geometric lattices," J. Combin. Theory, Series A 38 (1985), 66-74.

27. W.M. Kantor, "Primitive permutation groups of odd degree, and an application to finite projective planes," J. Algebra 106 (1987), 15-45.

28. W.M. Kantor, "2-transitive and flag-transitive designs," in D. Jungnickel et al. (Eds.), Coding Theory, Design Theory, Group Theory, Proc. Marshall Hall Conf. (Burlington, VT, 1990), J. Wiley, New York (1993), pp. 13-30.

29. P.B. Kleidman and M.W. Liebeck, "The Subgroup Structure of the Finite Classical Groups," London Math. Soc. Lecture Note Series 129, Cambridge Univ. Press, Cambridge, 1990.

30. P.B. Kleidman, "The finite flag-transitive linear spaces with an exceptional automorphism group," in E.S. Kramer and S.S. Magliveras (Eds.), Finite Geometries and Combinatorial Designs (Lincoln, NE, 1987), Contemp. Math. 111, Amer. Math. Soc., Providence, RI (1990), pp. 117-136.

31. M.W. Liebeck, "The affine permutation groups of rank three," Proc. London Math. Soc. 54(3) (1987), 477-516.

32. M.W. Liebeck, "The classification of finite linear spaces with flag-transitive automorphism groups of affine type," J. Combin. Theory, Series A 84 (1998), 196-235.

33. H. Lüneburg, "Fahnenhomogene Quadrupelsysteme," Math. Z. 89 (1965), 82-90.

34. E. Maillet, "Sur les isomorphes holoédriques et transitifs des groupes symétriques ou alternés," J. Math. Pures Appl. 1(5) (1895), 5-34.

35. J. Saxl, "On finite linear spaces with almost simple flag-transitive automorphism groups," J. Combin. Theory, Series A 100 (2002), 322-348.

36. M. Suzuki, "On a class of doubly transitive groups," Ann. Math. 75(2) (1962), 105-145.

37. J. Tits, "Sur les systèmes de Steiner associés aux trois "grands" groupes de Mathieu," Rendic. Math. 23 (1964), 166-184.

38. H.N. Ward, "On Ree's series of simple groups," Trans. Amer. Math. Soc. 121 (1966), 62-89.

39. E. Witt, "Die 5-fach transitiven Gruppen von Mathieu," Abh. Math. Sem. Univ. Hamburg 12 (1938), 256-264.

40. E. Witt, "Über Steinersche Systeme," Abh. Math. Sem. Univ. Hamburg 12 (1938), 265-275. 